\title{
Oesophageal injury suspected to be due to doxycycline ingestion
}

\author{
A N R Fernandopulle ${ }^{1}$, N M M Navarathne ${ }^{1}$
}

\begin{abstract}
Doxycycline induced oesophageal injury (DIEI) has contributed to $27 \%$ of the global burden of drug induced oesophageal injury. We report 21 cases of DIEI detected over a one year period.

Cases were defined as sudden onset - chest pain, dysphagia and odynophagia within a few hours to days after taking doxycycline, confirmed by UGIE, with improvement on withdrawal of doxycycline. The majority was in the 20 - 30 year age group and was female. The commonest symptoms were dysphagia and odynophagia. The mean time to symptoms from ingestion of doxycycline was 2.5 days. Majority of ulcers were in the mid oesophagus. Our case series highlights a preventable adverse drug reaction, which may have been avoided by giving simple advice to the patient.
\end{abstract}

Ceylon Medical Journal 2011; 56: 162-163

\section{Introduction}

Drug induced oesophageal injury is a well known adverse drug reaction with over 1000 cases caused by almost 100 different drugs which have been reported in the world literature upto 1999 [1, 2]. Although impressive, the numbers are considered by experts in the field to be quite small compared with the estimated case frequency [1]. Doxycycline induced oesophageal injury (DIEI) has contributed to $27 \%$ of the global burden [3]. No cases have been reported to date to the National Pharmacovigilance Unit in Sri Lanka, which limits the ability to assess the epidemiology of this iatrogenic disease as doxycycline is used commonly in our country. We report 21 cases of doxycycline induced oesophageal injury in an attempt to increase its awareness and possibly reduce its occurrence. This case series is timely as doxycycline is recommended on a weekly dosage regime in the national chemoprophylaxis programme for leptospirosis.

\section{Methods}

All cases referred for upper gastrointestinal endoscopy (UGIE) with suspected DIEI from January 2010 to December 2010 were evaluated. Cases were defined as sudden-onset chest pain, dysphagia and odynophagia within a few hours to days after taking doxycyline, confirmed by UGIE within a week of referral, with improvement on withdrawal of doxycycline. Cases were excluded if date of starting therapy, date of symptoms, and dosage regime were not recorded, and did not report for follow up.

\section{Results}

Twenty one cases fulfilled the selection criteria. Their age ranged from 13 to 41 years (mode: 20-30 years) and $76 \%$ were females. The commonest symptom was dysphagia (19/21) followed by odynophagia (18/21) and chest discomfort (10/21). The mean time to symptoms from ingestion of doxycycline was 2.5 days (range 1-5 days). All had ingested capsules. UGIE revealed localised ulcers at the mid, upper and lower oesophagus in $81 \%, 14 \%$ and $5 \%$ respectively. The ulcers were variable in size, shape and depth (Figure 1). No bleeding or significant strictures were seen. Doxycycline was withdrawn and all improved within 7 to 14 (median 10) days.

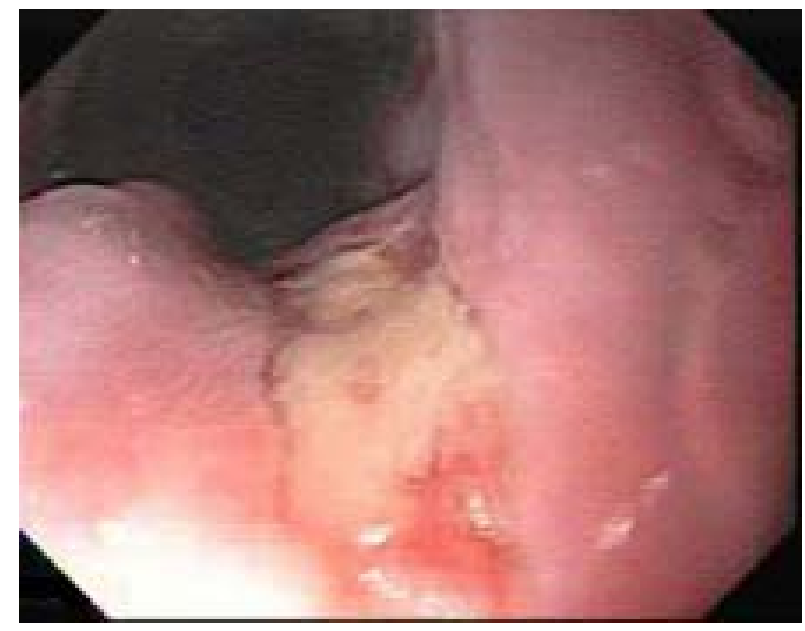

Figure 1.

\section{Discussion}

Doxycycline-induced oesophageal ulceration has been reported mostly in young persons with no previous history of oesophageal dysfunction which is compatible with our case series where the ages ranged from 13-44

${ }^{1}$ Gastroenterology and Hepatology Unit, National Hospital of Sri Lanka.

Correspondence: NMMN, e-mail address: <nmmnawarathne@gmail.com>. Received 22 June and revised version accepted 29 September 2011. Competing interests: none declared. 
years. The timing of symptoms was also in agreement with other studies where symptoms, usually, manifested within a few hours up to ten days. Sudden odynophagia is the most common presenting symptom documented [2]. Despite the absence of strictures, dysphagia was the commonest in our case series which is similar to the findings in Saudi Arabia [4]. Symptoms typically get better within a few days of discontinuing doxycycline as was seen in our cases but protracted courses up to six weeks have been reported [5].

Although the typical history is sufficient to establish the diagnosis, endoscopy remains the gold standard for causality assessment. Our UGIE findings are similar to other studies where tetracycline tended to cause distal oesophagitis whereas doxycycline frequently caused midoesophageal ulceration [6]. Lower oesophageal ulcers have been infrequently observed as in one of our cases.

Drug and patient factors play a role in DIEI. As an acidic drug, doxycycline accumulation in the epithelial cells can cause a focal contact oesophagitis. With its subsequent local cytochemical effects, doxycycline can cause ulceration and friability of the adjacent oesophageal mucosa. In addition, doxycycline can also inhibit protein synthesis functions in the oesophagus [7]. None in our study had the high risk patient factors for DIEI such as pre-existing oesophageal diseases such as reflux oesophagitis, scleroderma or oesophageal motility disorders.

Taking high risk drugs with little water predisposes to retention within the oesophagus and facilitates mucosal injury. Moreover, bedtime pill taking may favour retention of the pill within the oesophagus, because in the supine position, effect of the gravity disappears and there is decreased salivation and swallowing during sleep [1]. High risk drugs include tetracycline, doxycycline, quinidine, potassium chloride and alendronate.

In conclusion, our case series highlights two important facts. Firstly, under-reporting of DIEI, which may have led to inadequate awareness and education on a preventable ADR. Secondly, that young and healthy individuals are not immune to drug induced injury. Giving simple advice on swallowing the drug with a full glass of water and avoidance of lying down for at least 30 minutes after taking the medications would prevent unpleasant complications and unnecessary health expenditure on preventable medication errors.

\section{References}

1. Pacea F, Antinorib S, Repici. What is new in esophageal injury (infection, drug-induced, caustic, stricture, perforation). In: Tytgat GNJ eds. A Current Opinion in Gastroenterology. Lippincott Williams \& Wilkins. Inc. 2009; 25: 372-9.

2. Kikendall JW. Pill esophagitis. Journal of Clinical Gastroenterology 1999; 28: 298-305.

3. Al-Mofarreh MA, Al Mofleh IA. Esophageal ulceration complicating doxycycline therapy. World Journal of Gastroenterology 2003; 9: 609-11.

4. Tankurt IE, Akbaylar H, Yenicerioglu Y, et al. Severe longlasting symptoms from doxycycline-induced esophageal injury. Endoscopy 1995; 27: 626.

5. Gencosmanoglu R, Kurtkaya-Yapicier O, Tiftikci A, et al. Mid-esophageal ulceration and candidiasis-associated distal esophagitis as two distinct clinical patterns of tetracycline or doxycycline-induced esophageal injury. Journal of Clinical Gastroenterology 2004; 38: 484-9.

6. Kikendall JW, Friedman AC, Oyewole MA, et al. Pillinduced esophageal injury. Case reports and review of the medical literature. Digestive Diseases and Sciences 1983; 28: $174-82$.

7. Baehr PH, McDonald GB. Esophageal disorders caused by infection, systemic illness, medications, radiation, and trauma. In: Feldman M, Scharschmidt BF, Sleisenger MH, eds. Gastrointestinal and Liver Disease. 6th ed. WB Saunders: Philadelphia, 1998: 519-39. 